\title{
Teriparatide in the management of osteoporosis
}

\author{
Donald Bodenner \\ Carolyn Redman \\ Ann Riggs
}

Department of Geriatrics, University of Arkansas for Medical Sciences, Little Rock, AR, USA
Correspondence: Donald Bodenner University of Arkansas for Medical Sciences, Box 806, 430I West Markham Street, Little Rock, AR 72205, USA

Tel + I $50 \mid 5265705$

$\mathrm{Fax}+\mid$ 50I 5265710

Email Bodennerdonald@uams.edu

\begin{abstract}
Fracture of the hip is frequently a catastrophic event in the elderly, often resulting in death within a year and of the survivors, few regain pre-fracture quality of life. Although less appreciated, fractures of the spine result in significant morbidity and are also associated with increased mortality compared with individuals without a fracture. In recent years there has been an explosion in the development of new drugs for the treatment of osteoporosis. Recombinant human parathyroid hormone (1-34) $(20 \mu \mathrm{g} /$ day) is a recent addition to this armamentarium with a novel mechanism of action, which was approved by the US FDA for the treatment of postmenopausal osteoporosis and male osteoporosis secondary to hypogonadism in November 2002. It is the first osteoporosis treatment that leads to the formation of new bone with architecture similar to normal bone. Intense efforts have been made to understand the effect of teriparatide on antiresorptive therapy and vice versa. Although these relationships are not completely understood, the results of recent studies allow clinicians to begin to optimize therapeutic gains in bone mineral density and improve anti-fracture efficacy.
\end{abstract}

Keywords: osteoporosis, teriparatide, fracture

\section{Introduction}

There are more than 1.5 million fractures each year that are attributed to osteoporosis in America at a cost of US\$14 billion to the US health care system (NOF 2007). One in two women and one in four men over the age of 50 will suffer a fracture related to osteoporosis (NOF 2007), with absolute risk of fracture increasing two fold for each decade of life (Siris et al 2006). While fractures of the hip and vertebrae are most frequently sited, non-hip, and non-vertebral fractures account for a significant proportion of fractures and are also associated with significant morbidity (Delmas et al 2007). Over $20 \%$ of persons aged 50 and older with hip fracture die within the first year. Death rates are higher in men, often exceeding $30 \%$, and increase with advancing age (Cauley et al 2000). Hip fracture leads to loss of functional independence, with approximately one-third of previously independent individuals requiring nursing home placement, and at 6 months, only $15 \%$ regain ambulatory status without an assistive device (NOF 2007). Up to $60 \%$ of individuals will require some assistance a year later (IOF 2007). Vertebral fractures account for approximately $45 \%$ of osteoporosis-related fractures, and have significant immediate and long-term complications such as pain, respiratory complications, loss of height, and loss of quality of life. Of those presenting with vertebral fractures, the risk of new vertebral and non-vertebral fracture increases 4- to 5-fold (Klotzbuecher et al 2000). Moreover, there is mounting evidence that vertebral fractures also increase mortality within 1 year of the fracture and this excess risk increases with time (Cooper et al 1993; Center et al 1999; Cauley et al 2000). Clearly osteoporosis is a major public health concern with significant health care expenditures and personal burden. As the baby boomers enter retirement age, the percentage of elder adults will increase. The burden of osteoporosis related fractures is projected to reach over 3 million fractures per year with substantial increases in health care expenditures estimated at over US\$25.3 billion per year (Burge et al 2007). 


\section{Fracture risk}

Although great strides have been made in identifying and treating individuals with osteoporosis, osteoporosis related fractures remain a major public health problem. Osteoporosis related fractures occur with little or no trauma and are often called fragility fractures. Up to $40 \%$ of white women and $13 \%$ of white men in the US will suffer at least one fragility fracture in their lifetime (IOF 2007; NOF 2007). Although low bone mineral density (BMD) is highly predictive of an increased risk of future fracture (WHO 1994), multiple factors are involved in estimating an individual's risk including age, race, previous fracture, glucocorticoid use, gender, history of falling, hyperparathyroidism, and malabsorption syndromes. It is important to recognize these risk factors, for they can have a profound effect on the estimation of overall risk. For instance whites have twice the fracture risk of Asians and blacks (Barrett-Connor et al 2005), and women have almost twice the rate of fracture as men at any site (van Staa et al 2001). The risk of a second vertebral fracture in an individual with a previous vertebral fracture is at least 4-fold (Klotzbuecher et al 2000) and the risk of hip fracture is doubled compared with those fracture free (Kanis et al 2004). Despite this clear predictor of increased risk, only one-third of all vertebral fractures noted on radiographs come to medical attention (Cooper et al 1992). Age is a strong risk factor independent of BMD, with a 70-year-old female having double the risk of fracture compared with a 50-year-old female with the same BMD (Kanis et al 2004). Frailty is also an independent risk factor; individuals with a high fall risk and low BMD were at least 4-fold more likely to fracture than women without low BMD or falls (Dargent-Molina et al 1996). These and other studies indicate that BMD is crucial in the evaluation for osteoporosis as an initial step, but other risk factors must be identified that may modify treatment or change the decision to treat. For instance, a vertebral fragility fracture indicates a high risk of future fracture regardless of BMD and therapy is indicated.

\section{Mechanism of action}

Osteoporosis develops when there is an imbalance between bone formation and bone resorption. In states of high turnover, such as sex steroid deficiency, rheumatoid arthritis, and certain malignancies, resorption occurs in excess of formation. Antiresorptive agents such as estrogen, raloxifene, bisphosphonates, and calcitonin inhibit both aspects of bone remodeling, but slow resorption more than formation. Teriparatide is the first approved anabolic, or bone building drug, where bone formation is stimulated more than resorption. To clinicians this seems counterintuitive, because the elevated levels of parathyroid hormone (PTH) found in hyperparathyroidism typically result in reduced BMD and increased fracture risk. Research has shown that bone formation occurs soon after PTH is administered because osteoblast formation is increased and osteoblast apoptosis is inhibited, which results in an increase in bone turnover and formation (Lindsay et al 1997).

Therefore, intermittent administration increases the number of bone forming osteoblasts whereas continuous administration increases the number of bone resorbing osteoclasts (Dobnig et al 2005). Overall there is a net increase in bone with each PTH cycle (Dobnig and Turner 1997; Hagino et al 2001). Importantly, the bone formed is "good" bone with histomorphometry and peripheral quantitative $\mathrm{CT}$ (pqCT) showing an increase in trabecular width and number (Figure 1), structurally similar to bone from younger individuals (Jiang et al 2003). This remodeling predominantly occurs at bone surfaces subject to mechanical stress (Hagino et al 2001; Hodsman et al 2005).

\section{Clinical response to teriparatide as single therapy}

The largest number of teriparatide treated patients was examined in a phase III clinical trial published in 2001 (Neer et al 2001). Over 1600 postmenopausal women with prior vertebral fractures were randomized to receive placebo or $20 \mu \mathrm{g}$ or $40 \mu \mathrm{g}$ of teriparatide for a median of 21 months. Compared with placebo, teriparatide increased BMD at the lumbar spine by $9 \%$ and $13 \%$, respectively, and at the femoral neck by $2 \%$ to $5 \%$ over placebo $(\mathrm{p}<0.001)$. More importantly, the reduction in new vertebral fractures compared with control was highly significant and similar between the two doses $(65 \%$ and $69 \%$ respectively, $\mathrm{p}<0.001)$ (Figure 2 ). The reduction in the subset of vertebral fractures judged to be moderate to severe by radiologic criteria was even more pronounced, approximately $90 \%$ in both treatment groups (Figure 2). Fragility fractures at nonvertebral sites such as the hip and wrist were reduced by $53 \%(\mathrm{p}<0.05)$. This study represents the effect of teriparatide alone, for the number of osteoporosis treatment-naive patients were similar between the placebo and treatment groups ( $85 \%$ vs $86 \%)$ and subjects with prior osteoporosis treatment were off therapy for at least 2 months prior to randomization. BMD at the wrist was reduced slightly in the teriparatide group compared with controls; however, fracture risk was halved. This decrease in BMD has been noted in other, smaller studies (Body et al 2002; Orwoll et al 2003; Kurland et al 2004). The explanation 

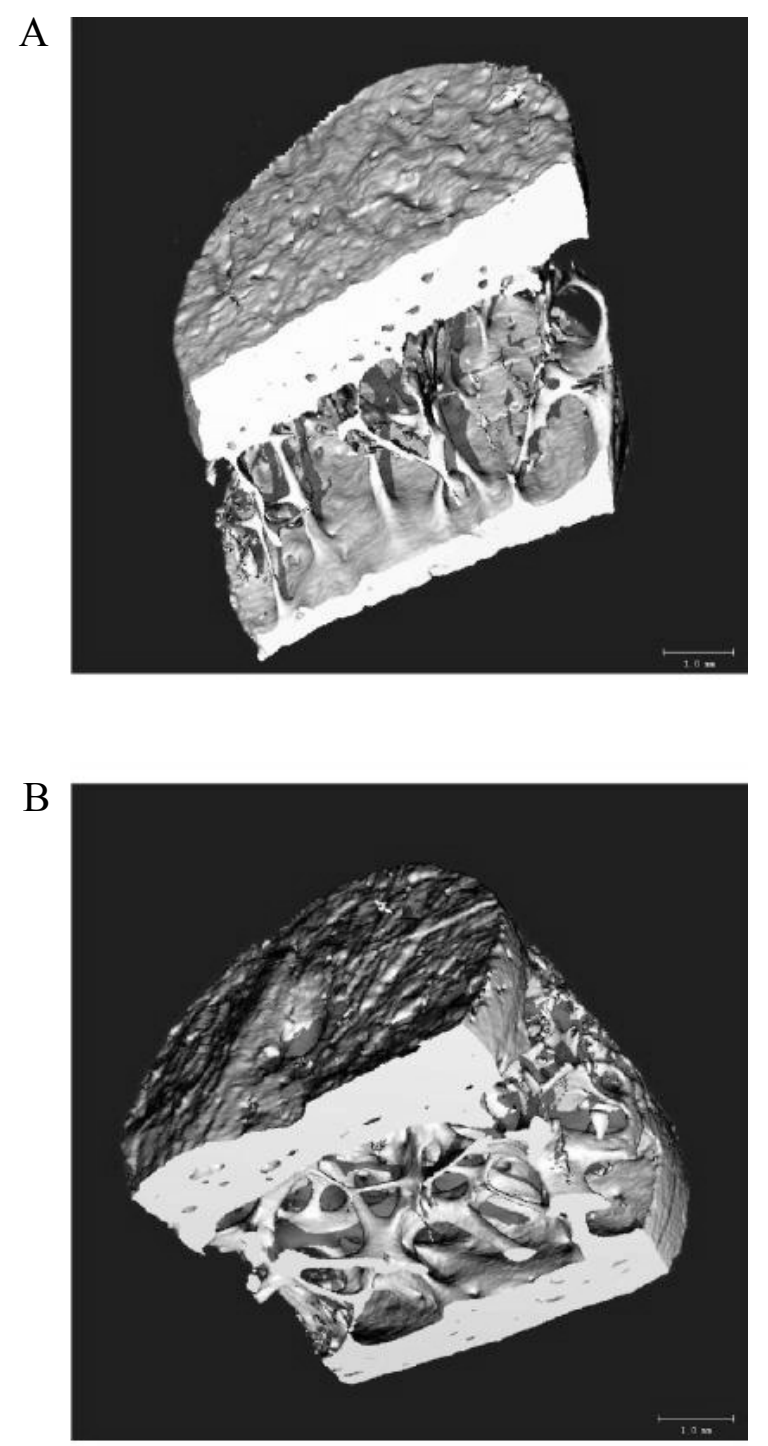

Figure I Teriparatide therapy improves skeletal architecture. Micro-CT scans of iliac crest biopsy specimens at baseline (A) and after 21 months of therapy (B) with teriparatide $(20 \mu \mathrm{g} / \mathrm{d})$. Reproduced with permission from Jiang Y, Zhao JJ, Mitlak BH et al 2003. Recombinant human parathyroid hormone (I-34) [teriparatide] improves both cortical and cancellous bone structure.J Bone Miner Res, 18:1932-4I. Copyright (C) 2003 American Society for Bone and Mineral Research.

for the discrepancy between decrease in BMD and decrease in fracture risk at the wrist is unclear. One explanation may be that increased cortical deposition and remodeling result in decreased bone density but increased bone diameter resulting in greater bone strength.

A comparison between the effects of teriparatide and other treatments on new vertebral fractures and nonvertebral fractures is shown in Table 1. Although teriparatide decreased fractures at both vertebral and non-vertebral sites more than most other treatments, these are not head-to-head studies of these agents. Direct comparisons cannot be made because of differences in study design and patient populations.
Nonetheless, the data show that teriparatide is at least as efficacious as other accepted therapies for osteoporosis.

\section{Whom should be treated?}

Much of the recent research into teriparatide has involved examining the effects of other osteoporosis therapies on the response to teriparatide. From a clinical standpoint, this may have overly complicated the approach to teriparatide use, for the initial decision is who should be treated. A first step in this process may be to consider whether the anabolic mechanism represented by teriparatide is inherently superior to the mechanism of antiresorptive therapy. In other words, in a perfect world where cost is not a consideration, should one class be chosen as first-line therapy over another? The definitive answer to this important question is not yet known, for there are insufficient teriparatide follow-up data. However, from what we know as discussed above, anabolic agents make new bone with increased trabecular number and thickness. Antiresorptive agents inhibit bone remodeling and prevent further bone loss. Increases in BMD do occur with antiresorptives, but this occurs by the filling of resorptive cavities and prolonging mineralization, not from an improvement in the inherent structure of bone. In humans, bone histomorphometry, and quantitative CT (qCT) show increased trabecular width and number after teriparatide treatment, with these changes structurally similar to bone from younger individuals (Hodsman et al 2005). If teriparatide actually improves bone structure, then why in the Phase III study were there continued fractures in the teriparatide treated group? The study had a median followup of 21 months from the initiation of therapy. The effects of teriparatide are not instantaneous and fractures would be expected early on while remodeling and advantageous changes in bone architecture occur.

Taken together, these considerations suggest that with the current clinical data and what we know of the mechanism of the two classes of drugs, anabolic therapy may be superior in some ways to antiresorptives. It should be stressed that there are no long-term clinical data at this time to support this conclusion, although for clinicians who find these arguments compelling, teriparatide appears to be an attractive first-line drug in certain individuals. However cost, ease of administration, and side-effects are, as always, significant considerations in the decision to treat. At the time of this writing, monthly treatment cost for teriparatide is approximately 3- to 4-fold that of bisphosphonates. Although not directly comparable, a cost-utility analysis performed in the UK between teriparatide, bisphosphonates, and raloxifene 
A

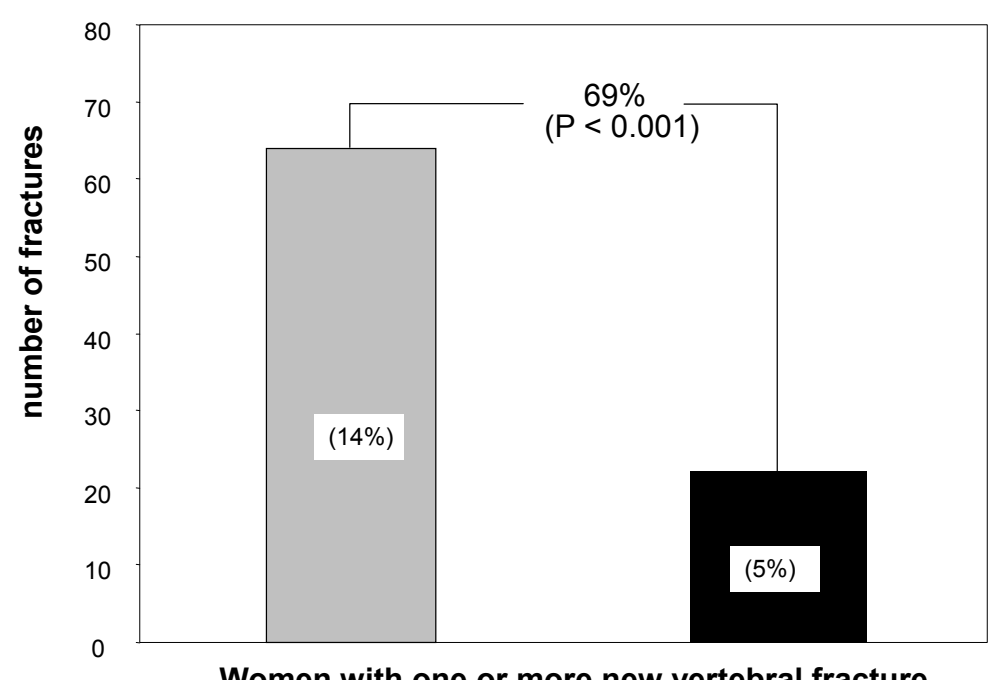

B

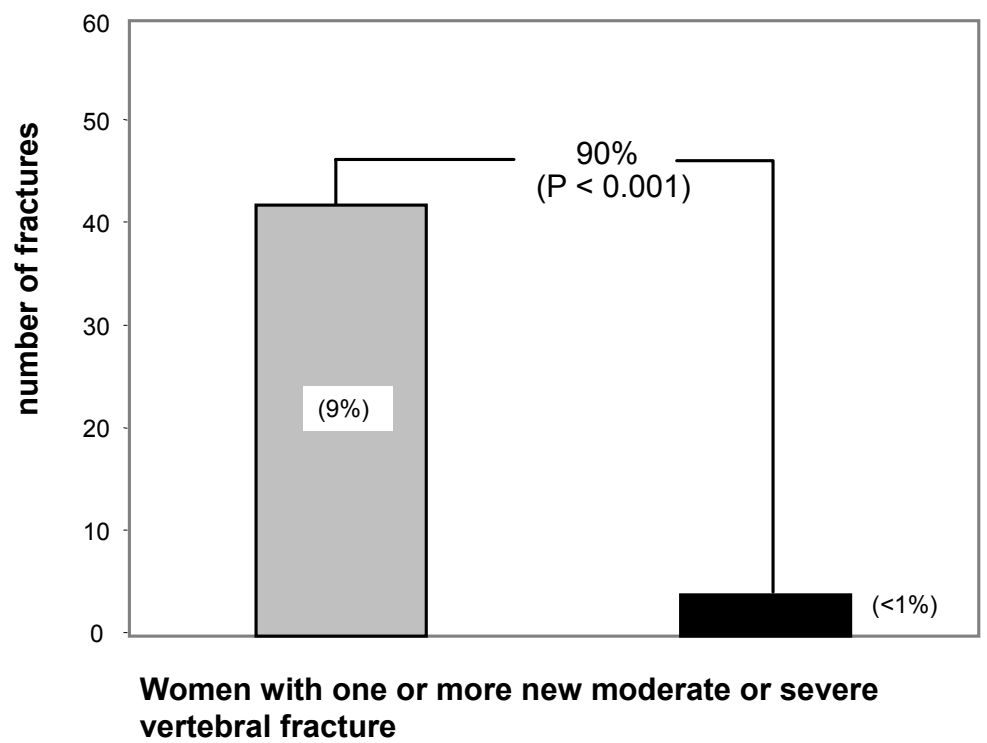

Figure 2 Effect of teriparitide treatment ( $20 \mu \mathrm{g} / \mathrm{d}$, black bar) compared with placebo (gey bar) on A. Risk of one or more new vertebral fracture. B. Risk of one or more new moderate or severe vertebral fractures.

showed that teriparatide treatment is justified only in patients with a 4-fold risk of fracture above the age-controlled baseline risk (NICE 2004). Moreover, teriparatide must be injected subcutaneously, which some patients find cumbersome and difficult. In terms of side-effects, as discussed in detail below, there are few side-effects associated with teriparatide treatment and subjects rarely withdrew from clinical trials.

It is important to stress that adequate calcium and vitamin D is crucial to ensure a good response to not only PTH, but other anti-osteoporosis agents. Abundant research suggests that up to $70 \%$ of the elderly suffer from hypovitaminosis D (Holick et al 2005; Mosekilde 2005). Agrawal et al studied the effectiveness of risedronate therapy in frail nursing home residents where $94 \%$ of the residents were vitamin D deficient (Agrawal et al 2006). They demonstrated that the expected drop in biomarkers of formation or resorption was not observed. Likewise, a preliminary report showed that vitamin D deficiency impaired the effect of alendronate in postmenopausal women (Ishijima et al 2005).

Teriparatide has been FDA approved for the treatment of patients at high risk of fracture, but assessing which patients 
Table I Therapeutic clinical trials evaluating fracture risk reduction in women with postmenopausal osteoporosis

\begin{tabular}{|c|c|c|c|c|c|c|}
\hline \multirow[t]{2}{*}{ Trial } & \multirow[t]{2}{*}{ Dose } & \multirow{2}{*}{$\begin{array}{l}\text { Mean } \\
\text { age }\end{array}$} & \multirow{2}{*}{$\begin{array}{l}\text { Treatment } \\
\text { duration }(y)\end{array}$} & \multicolumn{3}{|c|}{ Relative risk reduction (\%) } \\
\hline & & & & vertebral & $\begin{array}{l}\text { total } \\
\text { nonvertebral }\end{array}$ & hip \\
\hline \multicolumn{7}{|l|}{ Teriparatide } \\
\hline${ }^{\mathrm{b}} \mathrm{Neer}$ & $20-40 \mu \mathrm{g} / \mathrm{d}$ & 69 & 1.6 & 65 & 34 & \\
\hline \multicolumn{7}{|l|}{ Risedronate } \\
\hline \multirow[t]{2}{*}{ 'VERT-NA } & $5 \mathrm{mg} / \mathrm{d}$ & 69 & 1 & 65 & & \\
\hline & & & 3 & 41 & 39 & \\
\hline \multirow[t]{2}{*}{ DVERT-MN } & $5 \mathrm{mg} / \mathrm{d}$ & 71 & I & 61 & & \\
\hline & & & 3 & 49 & 33 (NS) & \\
\hline eHIP & $2.5-5 \mathrm{mg} / \mathrm{d}$ & 74 & 3 & & 20 & 40 \\
\hline \multicolumn{7}{|l|}{ Alendronate } \\
\hline \multirow[t]{2}{*}{ fFIT I } & $5-10 \mathrm{mg} / \mathrm{d}$ & 7I & 3 & 47 & 20 (NS) & 51 \\
\hline & & & & & & 21 \\
\hline gFIT 2 & $5-10 \mathrm{mg} / \mathrm{d}$ & 68 & 4 & 44 & $12(\mathrm{NS})$ & (NS) \\
\hline \multicolumn{7}{|l|}{ Ibandronate } \\
\hline "BONE & $2.5 \mathrm{mg} / \mathrm{d}$ & 69 & 3 & 62 & NS* & \\
\hline \multicolumn{7}{|l|}{ Raloxifene } \\
\hline MORE & $60 \mathrm{mg} / \mathrm{d}$ & 67 & 3 & 30 & NS & \\
\hline \multicolumn{7}{|c|}{ Hormone replacement } \\
\hline \multirow[t]{3}{*}{ WHI } & CEE 0.625 & & & & & \\
\hline & $\mathrm{mg} / \mathrm{d}$ plus & 63 & 5.6 & 35 & 25 & 33 \\
\hline & progestin & & & & & \\
\hline \multirow[t]{2}{*}{ kWHI } & CEE 0.625 & & & & & \\
\hline & $\mathrm{mg} / \mathrm{d}$ & 63 & 6.8 & 38 & & 39 \\
\hline \multicolumn{7}{|l|}{ Calcitonin } \\
\hline & & & & & & 50 \\
\hline IPROOF & $200 \mathrm{IU} / \mathrm{d}$ & 68 & 5 & 33 & 12 (NS) & (NS) \\
\hline
\end{tabular}

are at high risk is complex. Certainly patients over the age of 65 with markedly depressed $\mathrm{T}$ scores less than -3.5 fall into this category. This T score cutoff is somewhat arbitrary and the decision to treat elderly patients with teriparatide should also depend on other risk factors. Elderly patients with prior fragility fractures with BMD of $\mathrm{T}<2.5$ should also be considered for treatment. Also, patients who continue to fracture or lose BMD after two years on bisphosphonate therapy would fall into the high risk category (Hodsman et al 2005). More controversial would be patients under the age of 60 and elderly with less severe osteoporosis. Also, patients who are immobile and non-weight bearing may not experience the expected gains from teriparatide since mechanical loading regulates PTH-stimulated gains in new bone (Troen 2006).

Another important factor is compliance. Compliance in many studies of anti-osteoporosis agents was excellent with a low dropout rate, but in practice, long-term compliance has been disappointing. Poor compliance may be attributed to poor patient-caregiver understanding, a low level of motivation, and the fact that osteoporosis is a "silent" disease with no obvious manifestation until a fracture occurs (Cramer et al 2006).

A recent study demonstrated that the response to $\mathrm{PTH}$ is the same in the elderly compared with younger individuals (Boonen et al 2006). Patients from the original Fracture Prevention Trial (Neer et al 2001) were separated into two subgroups with patient age younger than 75 and 75 and older. No difference was noted between the groups with regard to bone turnover markers, femoral neck BMD, vertebral fractures, nonvertebral fragility fractures, height loss, hyperuricemia, or hypercalcemia.

\section{Effect of other drug treatments on teriparatide \\ Alendronate concurrently with PTH}

The effect of previous or concurrent treatment with bisphosphonates on teriparatide is unclear. Two studies were 
published concurrently in 2003 examining the effect of alendronate on PTH; one in postmenopausal women and one in elderly men with low bone density. In the first study, 238 postmenopausal women with $\mathrm{T}$ score less than -2.5 or less than -2.0 with at least one additional risk factor of osteoporosis were randomized to daily full length PTH (1-84), alendronate $(10 \mathrm{mg} / \mathrm{d})$, or both and followed for 12 months (Black et al 2003). The mean age of participants was 70 years in all groups and other clinical parameters were similar. No additive or synergistic effects on BMD were observed between the two therapies. The combination group showed BMD gains equal to but not greater than those obtained with PTH alone. Volumetric bone density using CT demonstrated a marked increase in the PTH alone group but combination therapy with PTH and alendronate was no different than alendronate alone. Biochemical markers of bone formation and resorption were depressed in the alendronate and combination group.

A similar study was conducted in 83 men with low BMD randomized to receive teriparatide (PTH 1-34), alendronate (10 mg daily) or both (Finkelstein et al 2003). In the combination group, teriparatide was begun after 6 months of alendronate therapy. BMD was not significantly different between alendronate and combination therapy at any site except the spine. PTH alone was superior to combination therapy at all sites except the total hip $(p=0.08)$. Biochemical markers of bone formation and resorption were markedly elevated in the PTH group, lower in the combination therapy group $(\mathrm{p}<0.01)$, and severely blunted in the alendronate alone group.

A third study examined the effect of daily teriparatide and treatment cycles of teriparatide administered every three months on elderly women with osteoporosis who had been taking alendronate for at least a year, and continued to take alendronate throughout the study (Cosman et al 2005). This study did not include a PTH alone group, so the effects of PTH on alendronate-treated patients cannot be directly assessed. The addition of PTH to alendronate resulted in significant increases in spinal BMD in both the daily and cyclic group ( $6.1 \%$ and $5.4 \%$ respectively). Although significant, these changes were less than that seen in most studies of PTH alone.

Taken together these studies demonstrate that with the bisphosphonate alendronate, combination with PTH results in increased bone turnover and BMD at the spine, but the increases are less than with PTH alone. There is no demonstrable additive or synergistic effect between alendronate and PTH therapy when used together.

\section{Alendronate before PTH}

A smaller study examined 59 postmenopausal women with BMD T scores $<-2.0$ who had received either alendronate or raloxifene for 18-36 months prior to treatment with teriparatide for 18 months (Ettinger et al 2004). BMD responded in the raloxifene group as if subjects had received PTH alone. Patients pretreated with alendronate, however, showed no change in BMD after 6 months, and then a gradual rise in BMD was observed. There are several limitations to this study. The number of patients in each group was small, but most notably there were no control groups for the raloxifene and alendronate groups. Also, changes in BMD were compared to historical controls.

\section{Alendronate following PTH}

In an extension to the intact PTH study discussed above, the effect of alendronate administration after 1 year of PTH (1-84) treatment was evaluated in 119 osteoporotic elderly women (mean age of 70 years) randomized to placebo (PTHplacebo) or alendronate at $10 \mathrm{mg} / \mathrm{d}$ (PTH-AL) for 1 additional year (Black et al 2005). The patient characteristics were again similar between the two groups with a mean age of 70 , similar age at menopause, and similar BMD at the hip, spine and radius. Over the entire two year span of the study, increase in spinal BMD was much greater in the PTH-AL group (12.1\%) than the PTH-control group $(4.1 \%, p=0.002)$. At the hip, PTH-AL was associated with a much larger increase in BMD than PTH-control $(p<0.001)$. There was significant loss of BMD at the wrist in both groups $(p<0.001)$. Looking specifically at the second year of the study, PTH-AL gained BMD at the spine (4.9\%) and the hip (3.6\%) compared with the PTH-control group where there was loss of BMD at the spine $(-1.7 \%)$ and no change at the hip. Markers of bone remodeling were elevated in the PTH-placebo and PTHalendronate group at 1 year, but both groups returned to baseline by 24 months.

These results suggest that alendronate therapy (and perhaps other bisphosphonates) administered after the recommended two year course of teriparatide would preserve and even extend the gains in BMD. This study was performed with the full-length PTH molecule but similar results would be expected with teriparatide.

\section{Summary of teriparatide in combination with alendronate}

Taken together, these studies suggest that alendronate therapy prior to, or concurrent with, PTH/teriparatide administration results in decreased bone remodeling and a blunted increase 
in BMD compared with PTH alone. This effect makes the timing of PTH initiation controversial. At least in one study, complete PTH effects on BMD are restored 6 months after alendronate has been discontinued (Ettinger et al 2004). Because teriparatide treatment is limited to 24 months in a patient's lifetime, bisphosphonate could be discontinued for 6 months prior to initiating PTH therapy to maximize response during this rather short treatment period. It has recently been shown that a 6-month discontinuation of alendronate therapy would not result in significant loss of BMD or increase in fracture risk (Black et al 2006). Alternatively, immediately starting PTH will afford some, albeit reduced benefit, and fracture risk may decrease sooner than increases in BMD secondary to beneficial remodeling.

BMD falls off following discontinuation of PTH, and this decrease can be abrogated by alendronate therapy. After a 2-year course of teriparatide, alendronate or other bisphosphonate therapy probably should be resumed.

There are several important caveats to these recommendations. First and foremost, there are no data on changes in fracture risk after combination therapy with alendronate and PTH. All of these studies are based on BMD as a surrogate for efficacy of therapy, and there is a poor correlation between the increase in BMD and decreased risk of fracture (Delmas and Seeman 2004). Second, intact PTH and teriparatide were used in these studies and their effects may not be interchangeable. Also, alendronate was the only bisphosphonate used in these studies. The effect of other bisphosphonates on teriparatide has not been examined. There are clear differences between bisphosphonates in terms of bone affinity (Leu et al 2006; Nancollas et al 2006). Perhaps other bisphosphonates may not blunt remodeling to the same degree as alendronate, with the possibility of concurrent bisphosphonate therapy or institution of PTH sooner after discontinuation of a bisphosphonate.

\section{Combination therapy with raloxifene and estrogen}

Raloxifene is a selective estrogen receptor modulator (SERM) with estrogen-like properties at the skeleton and lipid system and anti-estrogen effects at the uterus and breast. Raloxifene has anti-fracture efficacy at the spine similar to other antiresorptives. For the purposes of the present discussion, estrogen and raloxifene will be discussed together. An early study in the mid 1990s examined postmenopausal women on HRT randomized to receive placebo or teriparatide for 3 years. BMD steadily increased at the spine and hip reaching a maximum of $13 \%$ and $2.7 \%$ respectively (Lindsay et al
1997). A later study by the same group examined 52 postmenopausal women on HRT for at least 2 years that were again randomized to placebo vs teriparatide for 3 years. BMD at the spine increased $13.4 \%$ and at the hip 4.4\%. Biochemical markers of bone formation were increased with teriparatide treatment compared with placebo, and BMD and markers of bone formation did not substantially decrease for up to 1 year after discontinuation of teriparatide therapy (Cosman et al 2001).

The combination of raloxifene and teriparatide was examined as part of the comparison trial described above of 59 postmenopausal women who had received either alendronate or raloxifene for $18-36$ months prior to treatment with teriparatide for 18 months (Ettinger et al 2004). There was an unimpeded and highly significant increase in BMD in the group previously treated with raloxifene compared with baseline at the spine $(10.2 \%, \mathrm{p}<0.05)$ and the hip $(1.8 \%, \mathrm{p}<0.05)$.

In summary, it appears that prior or concurrent therapy with HRT or raloxifene does not inhibit the anabolic effects of PTH. These data suggest that addition of PTH will be beneficial under these circumstances. However, it is not known whether the combination affords additive or synergistic benefits.

\section{PTH and glucocorticoid-induced osteoporosis}

Treatment with glucocorticoids rapidly causes a decrease in BMD and increased fracture risk (van Staa et al 2002). Both risedronate and alendronate have been shown to substantially decrease the risk of fractures in glucocorticoid-induced osteoporosis (GIO), and treatment with these agents has become standard of care (Saag et al 1998; Eastell et al 2000). These patients often have very low BMD by the time treatment is initiated, and the use of an anabolic agent to build new bone would be an attractive alternative to bisphosphonate therapy or anabolic treatment antecedent to initiating a bisphosphonate. To evaluate PTH treatment of glucocorticoid-treated patients, 51 osteoporotic $(\mathrm{T}<-2.5)$ postmenopausal women receiving $8.0 \mathrm{mg}$ of prednisone per day $( \pm 3.78)$ and HRT were randomized to continued HRT and teriparatide for 1 year or continued HRT alone (Lane et al 1998; Rehman et al 2003). The mean age was 65 years and patients had been on HRT for approximately 16 years. In the PTH/HRT group, BMD as measured by quantitative CT and by DEXA had increased $35 \%$ and $11 \%$ respectively compared with the HRT alone group where BMD had increased only $1.3 \%$ and $0.2 \%$. A follow-up study for an additional year (24 months total) 
demonstrated that in the PTH/HRT group, BMD continued to increase at the vertebra measured by qCT and DXA ( $46 \%$ and $12.6 \%$ respectively) (Lane et al 2000). BMD also continued to increase at the hip attaining a $4.7 \%$ increase at the total hip and a 5.2\% increase at the femoral neck. There was no significant change in the HRT-alone group during the 12 months of additional follow-up.

It should be noted that teriparatide is not approved for the treatment of GIO, and this would constitute off-label use of the medication. Although these early results are promising, more studies are needed to evaluate the efficacy of PTH in younger patients and when used as a single agent. Nonetheless, in patients with GIO who do not respond to, or cannot tolerate bisphosphonates, $\mathrm{PTH}$ is an alternative worth consideration.

\section{Side-effects, exclusion criteria, and toxicity}

PTH injections, as one might expect, do have some effect on serum calcium levels. The phase III trial demonstrated that teriparatide levels reached a maximum within an hour and returned to baseline after 4 hours (Neer et al 2001). Serum calcium peaked approximately 4 hours after injection, but the increase was mild $(0.8 \mathrm{mg} / \mathrm{dL})$ and total calcium remained within the normal range in almost all subjects. Only $3 \%$ of patients required a calcium-based dose reduction in teriparatide. One-tenth of $1 \%$ of subjects withdrew because of increased calcium levels. Hypercalciuria was increased slightly, and $3 \%$ of patients had a significant increase in uric acid resulting in overt gout in several patients. Dizziness and leg cramps were associated with injection, and reported in $9 \%$ and $3 \%$ of patients respectively, statistically more than in the placebo group. Although the incidence of osteosarcoma was increased in animal trials involving rats, osteosarcoma has not been observed in human trials. The bone morphology changes induced in rats was highly abnormal, with gross overgrowth of bone almost to the obliteration of the marrow space (Vahle et al 2002). Nonetheless, the FDA placed a black box warning on the drug insert and all patients should be made aware of this prior to initiating therapy.

Teriparatide cannot be used in pediatric patients, patients who have Paget's disease, prior radiation to the bones, bone metastasis, pre-existing hypercalcemia, pregnancy, or metabolic bone disease other than osteoporosis.

\section{Conclusions}

Teriparatide is well-tolerated with few side effects, although daily subcutaneous injections and cost remain impediments to adoption in practice. There is substantial evidence that teriparatide functions as an anabolic agent with the induction of new bone formation that is structurally similar to normal bone. Teriparatide increases BMD, and reduces the risk of vertebral fracture and hip fracture. Concomitant or prior use of alendronate, but not raloxifene or estrogen, blunts the effects of teriparatide. The optimal alendronate holiday prior to instituting teriparatide is unknown, but probably will be at least 6 months. An antiresorptive should be added after a course of teriparatide is completed to prevent loss of BMD.

\section{References}

Agrawal S, Krueger DC, Engelke JA, et al. 2006. Between-meal risedronate does not alter bone turnover in nursing home residents. $J$ Am Geriatr Soc, 54:790-5.

Barrett-Connor E, Siris ES, Wehren LE, et al. 2005. Osteoporosis and fracture risk in women of different ethnic groups. J Bone Miner Res, 20:185-94.

Black DM, Bilezikian JP, Ensrud KE, et al. 2005. One year of alendronate after one year of parathyroid hormone (1-84) for osteoporosis. $N$ Engl J Med, 353:555-65.

Black DM, Greenspan SL, Ensrud KE, et al. 2003. The effects of parathyroid hormone and alendronate alone or in combination in postmenopausal osteoporosis. N Engl J Med, 349:1207-15.

Black DM, Schwartz AV, Ensrud KE, et al. 2006. Effects of continuing or stopping alendronate after 5 years of treatment: the Fracture Intervention Trial Long-term Extension (FLEX): a randomized trial. JAMA, 296:2927-38.

Body JJ, Gaich GA, Scheele WH, et al. 2002. A randomized double-blind trial to compare the efficacy of teriparatide [recombinant human parathyroid hormone (1-34)] with alendronate in postmenopausal women with osteoporosis. [see comment]. J Clin Endocrinol Metab, 87:4528-35.

Boonen S, Marin F, Mellstrom D, et al. 2006. Safety and efficacy of teriparatide in elderly women with established osteoporosis: bone anabolic therapy from a geriatric perspective. $J$ Am Geriatr Soc, 54:782-9.

Burge R, Dawson-Hughes B, Solomon DH, et al. 2007. Incidence and economic burden of osteoporosis-related fractures in the United States, 2005-2025. J Bone Miner Res, 22:465-75.

Cauley JA, Thompson DE, Ensrud KC, et al. 2000. Risk of mortality following clinical fractures. Osteoporos Int, 11:556-61.

Center JR, Nguyen TV, Schneider D, et al. 1999. Mortality after all major types of osteoporotic fracture in men and women: an observational study. Lancet, 353:878-82.

Cooper C, Atkinson EJ, Jacobsen SJ, et al. 1993. Population-based study of survival after osteoporotic fractures. Am J Epidemiol, 137:1001-5.

Cooper C, Atkinson EJ, O'Fallon WM, et al. 1992. Incidence of clinically diagnosed vertebral fractures: a population-based study in Rochester, Minnesota, 1985-1989. J Bone Miner Res, 7:221-7.

Cosman F, Nieves J, Woelfert L, et al. 2001. Parathyroid hormone added to established hormone therapy: effects on vertebral fracture and maintenance of bone mass after parathyroid hormone withdrawal. $J$ Bone Miner Res, 16(5):925-31.

Cosman F, Nieves J, Zion M, et al. 2005. Daily and cyclic parathyroid hormone in women receiving alendronate. $N$ Engl $J$ Med, 353(6):566-75.

Cramer JA, Lynch NO, Gaudin AF, et al. 2006. The effect of dosing frequency on compliance and persistence with bisphosphonate therapy in postmenopausal women: a comparison of studies in the United States, the United Kingdom, and France. Clin Ther, 28(10):1686-94. 
Dargent-Molina P, Favier F, Grandjean H, et al. 1996. Fall-related factors and risk of hip fracture: the EPIDOS prospective study. Lancet, 348(9021):145-9.

Delmas PD, Marin F, Marcus R, et al. 2007. Beyond hip: importance of other nonspinal fractures. Am J Med, 120(5):381-7.

Delmas PD, Seeman E. 2004. Changes in bone mineral density explain little of the reduction in vertebral or nonvertebral fracture risk with anti-resorptive therapy. Bone, 34(4):599-604.

Dobnig H, Sipos A, Jiang Y, et al. 2005. Early changes in biochemical markers of bone formation correlate with improvements in bone structure during teriparatide therapy. J Clin Endocrinol Metab, 90:3970-7.

Dobnig H, Turner RT. 1997. The effects of programmed administration of human parathyroid hormone fragment (1-34) on bone histomorphometry and serum chemistry in rats. Endocrinology, 138:4607-12.

Eastell R, Devogelaer JP, Peel NF, et al. 2000. Prevention of bone loss with risedronate in glucocorticoid-treated rheumatoid arthritis patients. Osteoporos Int, 11:331-7.

Ettinger B, San Martin J, Crans G, et al. 2004. Differential effects of teriparatide on $\mathrm{BMD}$ after treatment with raloxifene or alendronate. $J$ Bone Miner Res, 19:745-51.

Felsenberg D, Silman AJ, Lunt M, et al. 2002. The European Prospective Osteoporosis Study Group. Incidence of vertebral fracture in Europe: results from the European Prospective Osteoporosis Study (EPOS) J Bone Miner Res, 17:716-24.

Finkelstein JS, Hayes A, Hunzelman JL, et al. 2003. The effects of parathyroid hormone, alendronate, or both in men with osteoporosis. [see comment]. N Engl J Med, 349:1216-26.

Hagino H, Okano T, Akhter MP, et al. 2001. Effect of parathyroid hormone on cortical bone response to in vivo external loading of the rat tibia. J Bone Miner Metab, 19:244-50.

Hodsman AB, Bauer DC, Dempster DW, et al. 2005. Parathyroid hormone and teriparatide for the treatment of osteoporosis: a review of the evidence and suggested guidelines for its use. Endocr Rev, 26:688-703.

Holick MF, Siris ES, Binkley N, et al. 2005. Prevalence of Vitamin D inadequacy among postmenopausal North American women receiving osteoporosis therapy. J Clin Endocrinol Metab, 90:3215-24.

IOF 2007. Facts and statistics about osteoporosis and its impact. International Osteoporosis Foundation.

Ishijima M, Yamanaka M, Sakamoto Y. 2005. Vitamin D insufficiency impairs the effect of alendronate for the treatment of osteoporosis in postmenopausal women. J Bone Miner Res, 20(Suppl 1):S296.

Jiang Y, Zhao JJ, Mitlak BH, et al. 2003. Recombinant human parathyroid hormone (1-34) [teriparatide] improves both cortical and cancellous bone structure. J Bone Miner Res, 18:1932-41.

Kanis JA, Johnell O, De Laet C, et al. 2004. A meta-analysis of previous fracture and subsequent fracture risk. Bone, 35:375-82.

Klotzbuecher CM, Ross PD, Landsman PB, et al. 2000. Patients with prior fractures have an increased risk of future fractures: a summary of the literature and statistical synthesis. J Bone Miner Res, 15:721-39.

Kurland ES, Heller SL, Diamond B, et al. 2004. The importance of bisphosphonate therapy in maintaining bone mass in men after therapy with teriparatide [human parathyroid hormone (1-34)]. Osteoporos Int, 15:992-7.

Lane NE, Sanchez S, Modin GW, et al. 1998. Parathyroid hormone treatment can reverse corticosteroid-induced osteoporosis. Results of a randomized controlled clinical trial. J Clin Investig, 102:1627-33.
Lane NE, Sanchez S, Modin GW, et al. 2000. Bone mass continues to increase at the hip after parathyroid hormone treatment is discontinued in glucocorticoid-induced osteoporosis: results of a randomized controlled clinical trial. J Bone Miner Res, 15:944-51.

Leu CT, Luegmayr E, Freedman LP, et al. 2006. Relative binding affinities of bisphosphonates for human bone and relationship to antiresorptive efficacy. Bone, 38:628-36.

Lindsay R, Nieves J, Formica C, et al. 1997. Randomised controlled study of effect of parathyroid hormone on vertebral-bone mass and fracture incidence among postmenopausal women on oestrogen with osteoporosis. Lancet, 350:550-5.

Mosekilde L. 2005. Vitamin D and the elderly. Clin Endocrinol (Oxf), 62:265-81.

Nancollas GH, Tang R, Phipps RJ, et al. 2006. Novel insights into actions of bisphosphonates on bone: differences in interactions with hydroxyapatite. Bone, 38:617-27.

[NICE] National Institute for Clinical Excellence FA. 2004. The clinical effectiveness and cost effectiveness of technologies for the secondary prevention of osteoporotic fractures in postmenopausal women [online]. URL: www.nice.org.uk.

Neer RM, Arnaud CD, Zanchetta JR, et al. 2001. Effect of parathyroid hormone (1-34) on fractures and bone mineral density in postmenopausal women with osteoporosis. [see comment]. $N$ Engl J Med, 344:1434-41.

NOF 2007. Fast Facts on Osteoporosis. National Osteoporosis Foundation.

Orwoll ES, Scheele WH, Paul S, et al. 2003. The effect of teriparatide [human parathyroid hormone (1-34)] therapy on bone density in men with osteoporosis. J Bone Miner Res, 18:9-17.

Rehman Q, Lang TF, Arnaud CD, et al. 2003. Daily treatment with parathyroid hormone is associated with an increase in vertebral cross-sectional area in postmenopausal women with glucocorticoid-induced osteoporosis. Osteoporos Int, 14:77-81.

Saag KG, Emkey R, Schnitzer TJ, et al. 1998. Alendronate for the prevention and treatment of glucocorticoid-induced osteoporosis. Glucocorticoid-Induced Osteoporosis Intervention Study Group. N Engl J Med, 339:292-9.

Siris ES, Brenneman SK, Barrett-Connor E, et al. 2006. The effect of age and bone mineral density on the absolute, excess, and relative risk of fracture in postmenopausal women aged 50-99: results from the National Osteoporosis Risk Assessment (NORA). Osteoporos Int, 17:565-74.

Troen BR. 2006. Osteoporosis in older people:a tale of two studies (and three treatments). J Am Geriatr Soc, 54:853-5.

Vahle JL, Sato M, Long GG, et al. 2002. Skeletal changes in rats given daily subcutaneous injections of recombinant human parathyroid hormone (1-34) for 2 years and relevance to human safety. Toxicol Pathol, 30:312-21.

van Staa TP, Dennison EM, Leufkens HG, et al. 2001. Epidemiology of fractures in England and Wales. Bone, 29:517-22.

van Staa TP, Leufkens HG, Cooper C. 2002. The epidemiology of corticosteroid-induced osteoporosis: a meta-analysis. Osteoporos Int, 13:777-87.

[WHO] World Health Organization.1994. Assessment of fracture risk and its application to screening for postmenopausal osteoporosis. Report of a WHO Study Group. World Health Organ Tech Rep Ser, 843:1-129. 
